\title{
Notes on Eg'gs and Hatchlings of the Bengal Monitor (Varanus bengalensis)
}

Mehul Thakur

B-55, G.I.D.C. Colony, Pandesara, Surat, India 394221 (mehulnthakur@gmail.com)

Photographs by the author.

Tndia is home to four species of varanid lizards, the most geographically widespread being the Bengal Monitor (Varanus bengalensis), which occurs from southeastern Iran through much of southern Asia and into the Malay Archipelago (Pianka 2004). As in many wide-ranging varanids, the ecology and natural history of the species varies considerably throughout its range in response to different habitats and geoclimatic zones (Losos and Greene 1988; Auffenberg 1994; Pianka 1995).

The peak breeding period of Varanus bengalensis is January to April, but eggs have been found from June through December in fallen logs and termite mounds in Sri Lanka (Deraniyagala 1953). Monitors generally deposit their eggs in holes in the ground or in ant-hills (Smith 1935). Bengal Monitors lay their eggs in burrows excavated by the female if ant-hills or termitaria are not available (Auffenberg 1983). Clutch size varies according to the age of the female, with older animals laying more eggs than younger ones (Akond et al. 1982). Clutch sizes of 8-32 have been documented (Whitaker and Hikida 1981; Akond et al. 1982). Khan (1987) reported clutches of 20-30 eggs in Bangladesh and
Daniel (2002) recorded clutches of 8-30 in India. Eggs are oval and relatively soft-shelled (Smith 1935). The incubation period varies in response to temperature, humidity, and rainfall (Ahsan and Saeed 2004), with Daniel (2002) indicating a length of 8-9 months. Young monitors are much more brightly colored than adults (de Jong 1944). Hatchling Bengal Monitors are brown with distinct yellow dorsal spots that become increasingly indistinct in adults (Karunarathna et al. 2008).

On 18 October 2013, Prayas Team Environment received a distress call for a monitor in the water tank of a housing society in the Adajan area of Surat $\left(21.20309^{\circ} \mathrm{N}, 72.78355^{\circ} \mathrm{E}\right)$. Team member Bhavin Mistry found a female Bengal Monitor in the empty water tank with six eggs (Fig. 1). He swiftly bagged the lizard and collected the eggs. She subsequently laid two more eggs in the bag. The monitor was released near the rescue site. The eggs $(5.82 \pm 0.35 \mathrm{~cm}$ in length and $2.77 \pm$ $0.05 \mathrm{~cm}$ in width; mean \pm one std. dev.) were placed in loose soil at ambient temperatures. Hatchlings began to emerge after 198 days on 4 May 2014 (Fig. 1). Small slits were made in the eggs to facilitate emergence. Hatchlings were characteristi-
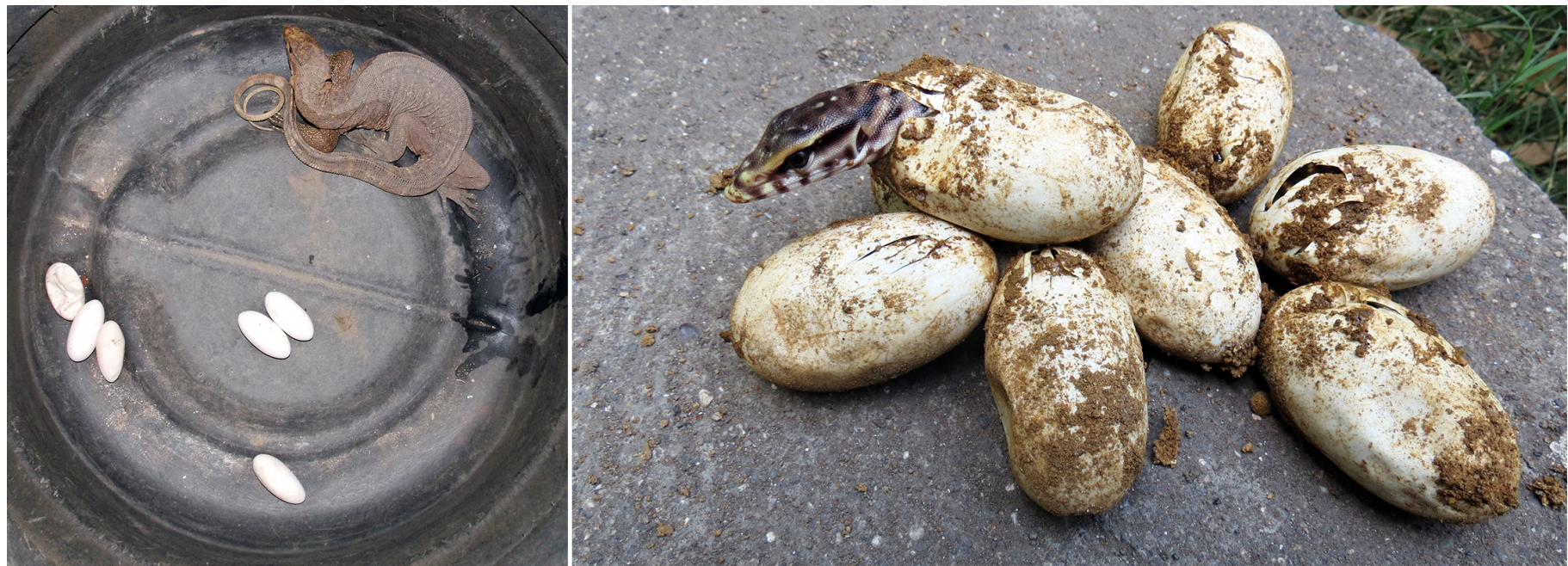

Fig. 1. Female Bengal Monitor (Varanus bengalensis) with eggs in a water tank at the rescue site (left). Hatchling Bengal Monitor emerging from an egg (right). 


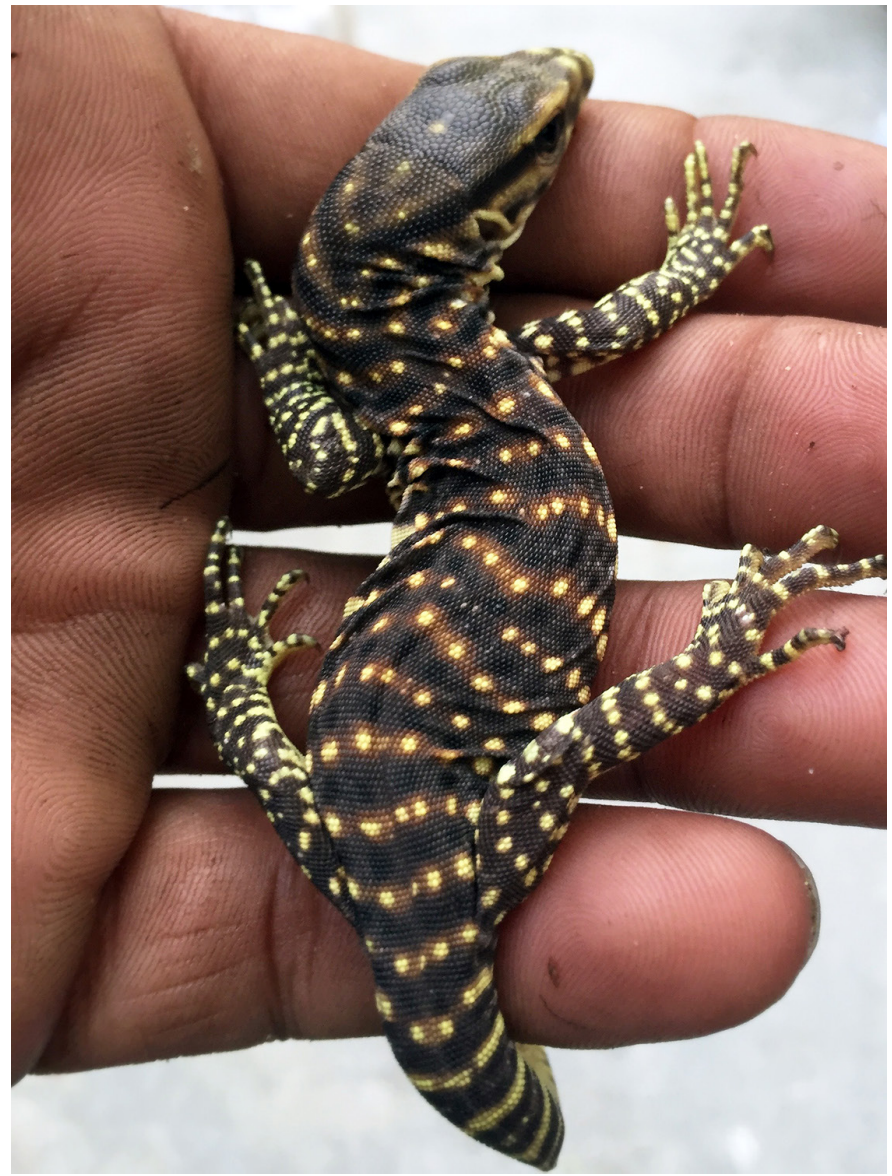

Fig. 2. Hatchling Bengal Monitor (Varanus bengalensis) prior to release.

cally brown in color with yellow dorsal spots. All were active and almost immediately began exhibiting defensive behaviors (hissing and elevating the head) when disturbed. All had an attached yolk sac, which provided nourishment for the first 2-3 days. The average total length of the six hatchlings was
$17.43 \pm 0.56 \mathrm{~cm}$. They were kept in captivity for four days and then released into suitable natural habitat (Fig. 2).

\section{Acknowledgements}

I am extremely grateful to Krunal Trivedi for his guidance and for providing literature. I also thank Prayas Team Environment and Bhavin Mistry for providing information on the rescue.

\section{Literature Cited}

Akond, A.W., F. Ahsan, and M. Rahman. 1982. Monitor lizards of Bangladesh, pp. 540-545. In: Proceedings of the Second Bangladesh National Conference on Forestry. 21-26 January 1982. Dhaka, Bangladesh.

Auffenberg, W. 1983. The burrows of Varanus bengalensis: Characteristics and use. Records of the Zoological Survey in India 80: 375-385.

Auffenberg, W. 1994. The Bengal Monitor. University Press of Florida, Gainesville, Florida.

Ahsan, M.F. and M.A. Safeed. 2004. Some aspects of breeding biology of the Bengal Monitor (Varanus bengalensis) in Bangladesh. Asiatic Herpetological Research 10: 236-240.

De Jong, J.K. 1944. Newly hatched Varanus komodoensis. Treubia 18: 143-145.

Deraniyagala, E.P.R. 1953. Tetrapod Reptiles of Ceylon. Volume 2. Government Press, Deraniyagala, Ceylon.

Daniel, J.C. 2002. The Book of Indian Reptiles \& Amphibians. Bombay Natural History Society and Oxford University Press, Mumbai, India.

Khan, A.R. 1987. Bangladesher Bonnya Prani, Part-1 (= Wildlife of Bangladesh, Part-1). Bangla Academy, Dhaka, Bangladesh.

Karunarathna, D.M.S.S., A.A.T. Amarsinghe, and A. de Vos. 2008. Preliminary notes on the monitor lizards (family: Varanidae) within the National Zoological Gardens (NZG) Dehiwala, Colombo District, Sri Lanka. Biawak 2: 109-118.

Losos, J.B. and H.W. Greene. 1988. Ecological and evolutionary implications of diet in monitor lizards. Biological Journal of the Linnean Society 35: 379-407.

Pianka, E.R. 1995. Evolution of body size: Varanid lizards as a model system. The American Naturalist 146: 398-414.

Pianka, E.R., D. King, and R.A. King. 2004. Varanoid Lizards of the World. Indiana University Press, Bloomington, Indiana.

Smith, M.A. 1935. The Fauna of British India Including Ceylon and Burma, Reptilia and Amphibia. Vol. II. - Sauria. Taylor and Francis, London, UK.

Whitaker, R. and J. Hikida. 1981. Unpublished report of the Project Formulation Mission to Bangladesh (Proceedings, 20 June-22 July 1981) to the Food and Agriculture Organization of the United Nations Action Programme for the Prevention of Food Losses, Rome, Italy. 